\title{
Teaching Manually Assisted Cough to Caregivers of Children With Neuromuscular Disease
}

\author{
Amelia F Kan, Jane M Butler DipPhyty GradDipAppSci MEd MTertEdMgt PhD, \\ Meghan Hutchence, Kristi Jones MBBS FRACP PhD CG (HGSA), John Widger MD FRACP, and \\ Michael A Doumit
}

BACKGROUND: Cough augmentation techniques are taught by health-care providers to improve secretion clearance and to help prevent respiratory infections in children with neuromuscular disease. There is some evidence of the effectiveness of a manually assisted cough when applied by health-care providers. However, it is unknown whether parents and caregivers may also be effective in applying manually assisted cough. The aim of this study was to evaluate whether parents and caregivers are effective at applying a manually assisted cough to a child with neuromuscular disease after being taught by a health-care provider. METHODS: For this prospective cohort study, children and their parents or caregivers were recruited from neuromuscular clinics in the Sydney Children's Hospitals Network. Cough peak flow was the outcome measure for the strength of the child's cough. Children were eligible to participate if their unassisted cough peak flow at baseline was $<270 \mathrm{~L} / \mathrm{min}$. Parents and caregivers were taught a manually assisted cough by a physiotherapist before being measured. The cough peak flow was measured in the following order: (1) during an unassisted cough as baseline, (2) during a manually assisted cough performed by a physiotherapist, (3) during a manually assisted cough performed by a parent or caregiver, and (4) during an unassisted cough after intervention. RESULTS: Twenty-eight children ( 24 boys, 4 girls; mean \pm SD age, $12 \pm 3$ y) completed the study. No clinically or statistically significant changes were found in the cough peak flow after the application of a manually assisted cough by parents or caregivers $(95 \% \mathrm{CI}-11$ to $11 \mathrm{~L} / \mathrm{min}$ ) or by physiotherapists $(95 \% \mathrm{CI}-6$ to $14 \mathrm{~L} / \mathrm{min})$. CONCLUSIONS: Parents and caregivers and health-care providers were ineffective at increasing cough peak flow in children with neuromuscular weakness when applying a manually assisted cough. A single training session was insufficient for a parent or caregiver to be able to apply a manually assisted cough effectively on his or her child with neuromuscular weakness. Further research is warranted to guide recommendations on how best to equip parents and caregivers with the skills to help manage children with neuromuscular disease. Key words: neuromuscular disease; cough augmentation; manually assisted cough; cough peak flow. [Respir Care 2018;63(12):1520-1527. (C) 2018 Daedalus Enterprises]

Introduction

Neuromuscular diseases (NMD) encompass an extensive range of debilitating and life-limiting conditions. ${ }^{1}$

\footnotetext{
Ms Kan and Dr Butler are affiliated with the School of Physiotherapy, Australian Catholic University, North Sydney, New South Wales, Australia. Ms Hutchence is affiliated with the Department of Physiotherapy, The Children's Hospital at Westmead, Westmead, New South Wales, Australia; Institute for Neuroscience and Muscle Research, Children's Hospital at Westmead, Westmead, New South Wales, Australia. Dr Jones is affiliated with the Disciplines of Genetics and Paediatrics, Sydney
}

These diseases affect neurons and impair communication between the brain and the muscles, which commonly leads to muscular, cardiac, and respiratory complications, ${ }^{2}$ and, consequently has profound impacts on an individual and
Medical School, University of Sydney, Sydney, New South Wales, Aus-
tralia; Department of Clinical Genetics, The Children's Hospital at West-
mead, Westmead, New South Wales, Australia; Neurogenetics/Neuro-
muscular Clinical Service, The Children's Hospital at Westmead,
Westmead, New South Wales, Australia; Clinical Trials Unit, Institute
for Neuroscience and Muscle Research, Children's Hospital at West-
mead, Westmead, New South Wales, Australia. Dr Widger is affiliated 
the family's health-related quality of life. ${ }^{3-5}$ Acute respiratory infections are of considerable concern for children with NMD because they are a major cause of unplanned hospital admissions. ${ }^{4}$ These admissions are caused by the cyclical nature of weakened respiratory muscles, secretion accumulation, infection, and chronic changes. Thus, clearing secretions is vital to reduce respiratory complications and, subsequently, improve survival and health-related quality of life..$^{3-5}$

Cough augmentation techniques are currently recommended to be taught to children when their cough peak flow falls below $270 \mathrm{~L} / \mathrm{min},{ }^{6}$ because this is the value at which it is likely that their cough peak flow will fall below $160 \mathrm{~L} / \mathrm{min}$ when acutely unwell, which is regarded as ineffective for secretion clearance. ${ }^{7}$ The rationale is that cough augmentation can be taught and then effectively used to aid secretion clearance when the child is unwell. Different health-care providers, depending on the healthcare system, may be involved with the training and administration of cough augmentation techniques. These health-care providers may include nurses, respiratory therapists, physiotherapists, and physicians.

A manually assisted cough is a cough augmentation technique in which an individual provides a thrust on the thoracic or abdominal region with his or her hands in coordination with the child's cough cycles, to enhance expiration. ${ }^{8}$ The rationale for use of a manually assisted cough is that vigorous compression of the chest simultaneously with expiration increases an individual's ex-

with the Department of Respiratory Medicine, Sydney Children's Hospital Network, Randwick, New South Wales, Australia; Discipline of Paediatrics, School of Women and Children's Health, University of New South Wales, Randwick, New South Wales, Australia. Mr Doumit is affiliated with the Department of Physiotherapy, Sydney Children's Hospital Network, Randwick, New South Wales, Australia; School of Women's and Children's Health, University of New South Wales, Randwick, New South Wales, Australia.

This study was performed at the Australian Catholic University in Sydney, New South Wales, Australia; Sydney Children's Hospital Network, Randwick, New South Wales, Australia; and The Children's Hospital at Westmead, Westmead, New South Wales, Australia.

Ms Kan presented a version of this paper at the Bachelor of Physiotherapy Honours Presentation Conference Day at Australian Catholic University, North Sydney, New South Wales, Australia, November 2, 2016, and at the Australian Physiotherapy Association Momentum Conference, October 21, 2017, Sydney, New South Wales, Australia.

The authors have disclosed no conflicts of interest.

Correspondence: Amelia F Kan, School of Physiotherapy, Australian Catholic University, North Sydney, New South Wales, Australia, 2060.

E-mail: amelia_kan@outlook.com.

DOI: $10.4187 /$ respcare.06213

\section{QUICK LOOK}

\section{Current knowledge}

Children with neuromuscular disease, their parents and caregivers are taught cough augmentation techniques to help clear secretions because acute respiratory infections are a major cause of hospitalization. A manually assisted cough is one cough augmentation technique that clinical studies have shown can be effective in children with neuromuscular disease when applied by a health-care provider. Currently, manually assisted coughs are not always taught as part of a child's neuromuscular disease home management program.

\section{What this paper contributes to our knowledge}

Manually assisted coughs were not effective when applied by parents or caregivers to a child with neuromuscular disease after being taught by a health-care provider in a single training session. Health-care providers may not be effective at applying a manually assisted cough to children with neuromuscular disease. This paper may guide clinical pediatric physiotherapy practice in how to best equip parents and caregivers with the skills to manage children with neuromuscular disease and how to use appropriate cough augmentation techniques for a child with neuromuscular disease.

piratory flow and thus can improve secretion displacement. ${ }^{9}$

When possible, parents should be involved in their child's respiratory management of NMD, and being taught cough augmentation techniques is part of this management so that this therapy can be provided in the home environment. However, to our knowledge there are currently no studies that have investigated a parent or caregiver's ability to effectively apply a manually assisted cough to his or her child. Therefore, there is little evidence guiding clinical practice on advising parents and caregivers in cough augmentation at home for children with NMD. Subsequently, the purpose of our study was to examine the ability of parents and caregivers to assist the child to improve the child's cough peak flow by using a manually assisted cough. Specifically, the aim of our study was to evaluate whether parents and caregivers of children with NMD were able to perform an effective manually assisted cough to the child after being taught by a health-care provider, as measured by an increase in the child's cough peak flow. We hypothesized that parents 
and caregivers would be effective in applying this technique.

\section{Methods}

\section{Subjects}

The study was a prospective cohort study approved by the Australian Catholic University Ethics Committee (2016-49HN) and the Sydney Children's Hospitals Network Ethics Committee (LNR/14/SCHN/553). Children and their parents and caregivers were prospectively recruited from neuromuscular clinics within the Sydney Children's Hospitals Network. Parents and caregivers signed informed consent for themselves and the children to participate. In addition, 2 physiotherapists (MAD and $\mathrm{MH}$ ) participated in the study, one at each hospital site. The physiotherapists were known to the children and to the parents and caregivers, and were experienced in the respiratory management of children with NMD. A child was included in the study if he or she was diagnosed with an NMD, attended a neuromuscular clinic within the Sydney Children's Hospitals Network, had a cough peak flow of $<270 \mathrm{~L} / \mathrm{min}$, and was between 6 and 18 y old. A child was excluded if he or she had contraindications that precluded the child from receiving manually assisted coughs or had severe cognitive and/or language deficits that precluded him or her from participating in the study.

\section{Outcome Measure}

Cough peak flow was the primary outcome measure used to measure a child's cough strength. Cough peak flow was measured during the expiratory phase of a cough by using an appropriately sized mask with a silicone seal (Ecomask, Intersurgical, Berkshire, United Kingdom) applied firmly to a child's face over the mouth and nose. The mask was connected to a low-range peak flow meter (Breath Alert, Allersearch, Victoria, Australia), and the strength of the cough was read and interpreted as $\mathrm{L} / \mathrm{min}$.

\section{Procedures}

Cough peak flow was measured under 4 different conditions for each child: (1) during a pre-intervention unassisted cough to establish the child's maximum unassisted cough peak flow for a baseline, (2) during a manually assisted cough performed by a physiotherapist, (3) during a manually assisted cough performed by a parent or caregiver, and (4) during a post-intervention unassisted cough. The interventions were not randomized but were applied in the order as mentioned. For each condition, the child was asked to breathe normally, take a maximal breath in, hold the breath as the mask was applied, and then cough with or without assistance. Three cough peak flow readings were recorded for each condition; in some cases, a child needed $>3$ attempts to ensure that 3 measures were within $10 \%$ of each other were recorded. The child was given at least a 30s rest between each cough and at least 2 min between conditions. For each child, all the measurements were taken with the child in the same position and environment, and the same investigator (AK) applied the mask. The same physiotherapist (MAD, MH) taught and applied the manually assisted coughs for each child.

\section{Pre-Intervention Unassisted Cough}

The pre-intervention unassisted cough measures were taken with the child in the same position in which the manually assisted cough was to be applied to establish the child's maximum unassisted cough peak flow. These measures were used as a baseline for a child's eligibility into the study and as a control for comparison with the other conditions.

\section{Manually Assisted Cough Performed by a Physiotherapist}

A physiotherapist (MAD, MH) selected the most appropriate manually assisted cough technique for each child based on his or her clinical experience with that child and the technique preference of the child. There were 4 possible alternative techniques (Fig. 1): (A) The child sitting upright, with the physiotherapist's hands around the bottom of the child's ribs; a push inward given as the child coughs; (B) the child sitting upright, with one of the physiotherapist's hands under the child's ribs and the other hand on the child's back to stabilize the child, a push up and inward is given as the child coughs; (C) the child is lying down or semi-reclined, with one of the physiotherapist's palms under the ribs of the child and the other arm over the ribs to stabilize the chest; and a push up and inward is given as the child coughs; and (D) the child is sitting upright or lying down, with the physiotherapist's hands on the child's ribs at either side of the child's chest, a push inward is given as the child coughs. In the upright sitting position, the child was seated in a chair or in his or her wheelchair, with the respective chair back against the wall. In the supine position, the child was positioned on a plinth, or in the semi-reclined position, the child was positioned in his or her wheelchair, with the backrest tilted.

\section{Manually Assisted Cough Performed by a Parent or Caregiver}

The physiotherapist (MAD, MH) taught the selected technique to the parent or caregiver of each child. Educa- 


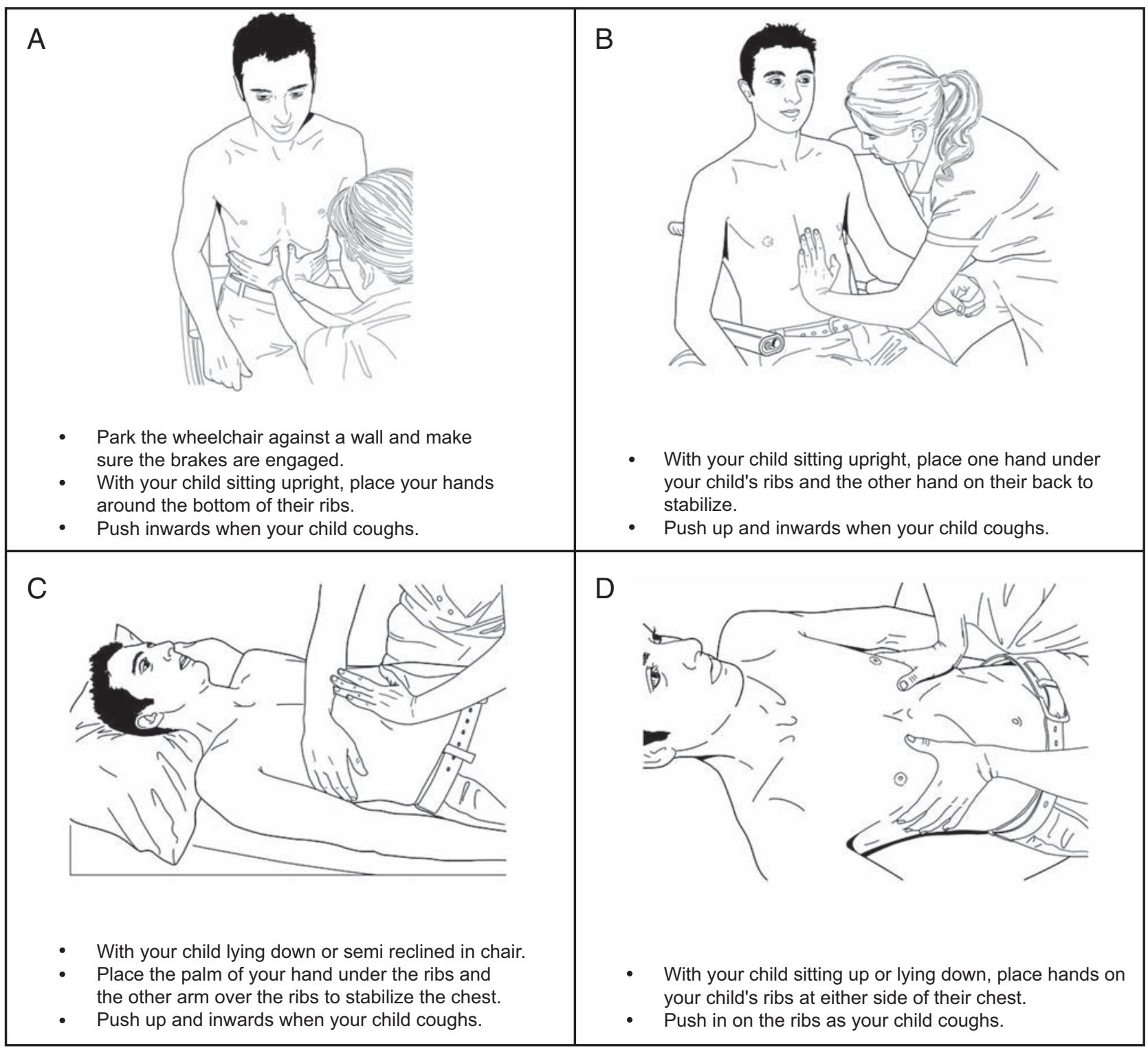

Fig. 1. Manually assisted cough technique alternatives in this study.

tion was given through demonstrations, manual guidance, and a handout. The physiotherapist also provided real-time verbal feedback between parent- or caregiver-assisted coughs. The parent or caregiver was allowed a maximum of 3 practice efforts before being measured.

\section{Post-Intervention Unassisted Cough}

The unassisted coughs measured after the intervention were taken to determine if a learning effect or fatigue may have influenced results, indicated by an increase or decrease, respectively, of the post-intervention cough peak flow in comparison with the baseline.

\section{Statistical Analysis}

The effectiveness of the parent's or caregiver's technique was measured by the change in cough peak flow during a parent- or caregiver-performed manually assisted cough compared with the pre-intervention baseline unassisted cough, and a physiotherapist (MAD, MH) performed manually assisted cough. These comparisons were analyzed by using parametric 2-tailed paired $t$ tests $^{10}$ from the highest of the cough peak flow values for each measured condition. Analysis was performed by using IBM SPSS Statistics 23 (SPSS, Chicago, Illinois), and statistical significance was regarded as a $P<.05$. Sample size was determined through a power analysis based 
Table 1. Group Characteristics of the Children With NMD

\begin{tabular}{lr}
\hline \hline \multicolumn{1}{c}{ Characteristics } & Children \\
& With NMD \\
\hline Age, mean \pm SD y & $12 \pm 3$ \\
Boys, $n$ (\%) & $24(86)$ \\
Pre-intervention unassisted CPF, mean \pm SD L/min & $181 \pm 61$ \\
Weight, mean \pm SD kg & $43 \pm 18$ \\
Weight, mean \pm SD Z score & $-0.5 \pm 1.5$ \\
Previously taught manually assisted cough, $n$ & 12 \\
Diagnosis, $n$ & \\
Centronuclear myopathy & 1 \\
Charcot-Marie-Tooth & 1 \\
Collagen 6 myopathy & 1 \\
Congenital myopathy & 1 \\
Duchenne muscular dystrophy & 12 \\
Facioscapulohumeral muscular dystrophy & 1 \\
Friedreich ataxia & 1 \\
Incomplete spinal cord injury & 1 \\
LMNA congenital muscular dystrophy & 1 \\
Nemaline myopathy & 3 \\
Unclassified NMD & 1 \\
Spinal muscular atrophy type 2 & 3 \\
Spinal muscular atrophy type 3 & 1 \\
\hline$N=28$. & \\
NMD = neuromuscular disease & \\
LPF = cough peak flow & \\
\hline & \\
\hline & \\
\hline &
\end{tabular}

on a previous study, ${ }^{11}$ which showed a difference in unassisted and assisted cough peak flow of $60 \mathrm{~L} / \mathrm{min}$ with a SD of 81 . Therefore, to detect an increase of $60 \mathrm{~L} / \mathrm{min}$ in the cough peak flow with $80 \%$ power, at a significance level of 0.05 , this study required at least 28 subjects.

\section{Results}

Thirty children and 30 respective parents or caregivers were invited to participate in the study. Two subjects withdrew from the study, both too fatigued during the baselineassisted cough attempts and, therefore, did not continue. Their results were not included in the analysis. Therefore, 28 children ( 24 boys and 4 girls; mean \pm SD age, $12 \pm 3$ y) and their respective parents or caregivers completed the study. Characteristics and baseline cough peak flow measurements for the children are presented in Table 1; the weight for one child was missing.

Cough peak flow did not significantly change from baseline when assisted by either physiotherapists or by parent or caregivers: pre-intervention unassisted cough (mean $\pm \mathrm{SD}$, $181 \pm 61 \mathrm{~L} / \mathrm{min}$ ), manually assisted cough performed by physiotherapists (mean $\pm \mathrm{SD}, 185 \pm 59 \mathrm{~L} / \mathrm{min}$ ), manually assisted cough performed by parents or caregivers (mean $\pm \mathrm{SD}, 181 \pm 63 \mathrm{~L} / \mathrm{min}$ ).
Of the 28 children who completed the study, the manually assisted cough technique applied was recorded for 21 of the children. The physiotherapists (MAD, MH) applied (Fig. 1) alternative A for 8 children, alternative B for 6 children, alternative $\mathrm{C}$ for 4 children, and alternative $\mathrm{D}$ for 3 children. The parents and caregivers applied alternative A for 9 children, alternative B for 4 children, alternative $\mathrm{C}$ for 3 children, and alternative $\mathrm{D}$ for 4 children. Three children had differing techniques used by the physiotherapist (MAD, MH) compared with the parent or caregiver. Data for the manually assisted cough alternatives used were missing for 7 of 28 children. We found no mean \pm SD difference at $0 \pm 15(95 \% \mathrm{CI}-6$ to 6$) \mathrm{L} / \mathrm{min}$ between pre-intervention unassisted cough peak flow at baseline and post-intervention unassisted cough peak flow, which indicated that there was not a learning or fatigue effect during the session (Table 2).

\section{Discussion}

To our knowledge, this was the first investigation that examined the effectiveness of parents and caregivers who administered a manually assisted cough to a child with NMD. The setting of this study was in Australian children's hospitals, where it is the physiotherapist's role to administer and teach manually assisted coughs. It is acknowledged that a range of health-care providers, including nurses, respiratory therapists, physiotherapists, and physicians, would have the role of administering and teaching cough augmentation. The profession of the health-care provider is determined by local practice in different healthcare systems. Therefore, even though physiotherapists were the health-care providers who participated in this study, the findings are applicable to all health-care providers who administer manually assisted coughs and provide respiratory care for children with NMD.

The main findings from this study revealed that parents and caregivers were unable to increase the child's cough peak flow by using a manually assisted cough alone after being taught by a health-care provider in a single training session. The findings from this study also indicated that health-care providers were not effective at applying a manually assisted cough to the children, as a mean $\pm \mathrm{SD}$ difference between the physiotherapist assistance versus the control of $4 \pm 27 \mathrm{~L} / \mathrm{min}$ can be considered negligible. No learning effect or fatigue was found in the children when pre- and post-intervention unassisted coughs were compared. Thus, with no fatigue, it is likely that the order in which the conditions were applied did not affect the results of the health-care providers' or parents' or caregivers' assistance.

The lack of increase in assisted cough peak flow was also evident in the narrow 95\% CI. In our study, when considering the effect of the parent- or caregiver-assisted 


\begin{tabular}{|c|c|c|c|}
\hline Paired Conditions & $\begin{array}{c}\text { Mean } \pm \mathrm{SD} \\
\text { Difference }(\mathrm{L} / \mathrm{min})\end{array}$ & $\begin{array}{c}95 \% \text { CI of Difference, } \\
\text { (L/min) }\end{array}$ & $P$ \\
\hline Physiotherapist-assisted cough minus pre-intervention unassisted cough & $4 \pm 27$ & -6 to 14 & .47 \\
\hline Parent- or caregiver-assisted cough minus pre-intervention unassisted cough & $0 \pm 28$ & -11 to 11 & .97 \\
\hline Physiotherapist-assisted cough minus parent- or caregiver-assisted cough & $4 \pm 20$ & -4 to 12 & .31 \\
\hline Pre-intervention unassisted cough minus post-intervention unassisted cough & $0 \pm 15$ & -6 to 6 & .95 \\
\hline
\end{tabular}

cough compared with the control, the $95 \%$ CI -11 to $11 \mathrm{~L} / \mathrm{min}$ showed that the true average effect of parent or caregiver assistance may be as high as $11 \mathrm{~L} / \mathrm{min}$ or as low as $-11 \mathrm{~L} / \mathrm{min}$, which is arguably too small to be of clinically important assistance or detriment given the pre-intervention unassisted cough peak flow (mean $\pm \mathrm{SD}$ $181 \pm 61 \mathrm{~L} / \mathrm{min}$ ). Similarly, the $95 \%$ CI -6 to $14 \mathrm{~L} / \mathrm{min}$ of physiotherapist assistance versus the control was narrow. Thus, although the effect of the parents or caregivers and health-care providers who apply a manually assisted cough was not likely to be clinically worthwhile, the application of a manually assisted cough by parents and caregivers and health-care providers will not be significantly detrimental to the child's cough strength.

Studies on the effectiveness of a manually assisted cough as a secretion clearance technique for children with NMD when performed by health-care providers showed varied results. Some studies indicated a large increase in cough peak flow, ${ }^{11-14}$ and other studies showed minor increases that are not likely to be clinically important. ${ }^{15-17}$ It should be noted that some of these studies looked at a manually assisted cough in combination with other cough augmentation techniques. ${ }^{12,14}$

The findings from some studies ${ }^{11-13}$ indicate a significant increase in cough peak flow when a health-care provider applies a manually assisted cough. Similar to our study, Sivasothy et $\mathrm{a}^{12}$ allowed a small number of practice coughs to familiarize participants with a manually assisted cough; however, the investigators found a statistically significant cough peak flow increase of $81 \mathrm{~L} / \mathrm{min}$ with a manually assisted cough application by a health-care provider compared with an unassisted cough. These results may have been due to a very small sample size, of 8 individuals who had neuromuscular weakness without a scoliosis. ${ }^{12}$ Interestingly, in the same study, it was shown that manually assisted coughs were not effective in individuals who had neuromuscular weakness and a scoliosis. ${ }^{12}$ Brito et $\mathrm{al}^{11}$ and Ishikawa et $\mathrm{al}^{13}$ showed significant increases, of $60 \mathrm{~L} / \mathrm{min}$ and of $66 \mathrm{~L} / \mathrm{min}$, respectively, in subjects with Duchenne muscular dystrophy. The differences in results between Ishikawa et $\mathrm{al}^{13}$ and our study may be related to the much lower unassisted cough peak flow, at mean $\pm \mathrm{SD} 138 \pm 70 \mathrm{~L} / \mathrm{min}$, found in their subjects. The investigators found that subjects with weaker coughs had larger increases in cough peak flow. ${ }^{13}$
Other studies indicate only a modest increase in cough peak flow when a health-care provider applies a manually assisted cough. Kang et $\mathrm{al}^{16}$ had a similar mean age group of $14 \mathrm{y}$, with a much larger sample size with results obtained from 51 participants but showed a modest increase, of $33 \mathrm{~L} / \mathrm{min}$, in cough peak flow compared with an unassisted cough peak flow. Kang et al ${ }^{15}$ and Chatwin et al ${ }^{17}$ found only modest increases of $35 \mathrm{~L} / \mathrm{min}$ and $19 \mathrm{~L} / \mathrm{min}$, respectively. Similar to our study, they had small sample sizes of 32 and 22 participants who had higher average ages, of 17 and $25 \mathrm{y}$, respectively.

Some elements of bias and limitations were present in our study. One potential source of bias was the lack of blinding of the investigators, physiotherapists, and subjects during data collection. Blinding could have been implemented during data collection via covering the readings on the cough peak flow meter. However, part of the study protocol involved training the parents to perform a manually assisted cough, and it was deemed necessary to provide real-time feedback on the effectiveness of the manually assisted cough being applied as part of the education process. Indeed, in other studies, blinding was either not mentioned or did not occur. ${ }^{12,13,16}$

Another source of bias was the limited standardization of alternative manually assisted cough techniques among the subjects. As identified in previous studies, manually assisted coughs have limitations, ${ }^{12,18}$ particularly when scoliosis or obesity is an issue. To accommodate for these limitations, the physiotherapists selected an appropriate technique for each child based on clinical judgment. Applying clinical judgment was consistent with other studies that also identified several manually assisted cough techniques to optimize each child's care. ${ }^{12,17}$ However, a selection of techniques meant that the intervention was difficult to standardize and, potentially, that biases resulted in a positive direction by accommodating for individual characteristics or, in a negative direction, because selection was based on clinical judgment rather than objective measurement. In the current study design, the parents and caregivers were encouraged to participate in the decision-making about the technique in which they felt most competent. Three of the parents or caregivers chose a different technique than that of the physiotherapist; however, the position of the child remained the same. Limited participant 
numbers meant that a valid comparison of the different types of manually assisted cough could not be made.

Measurement error bias may have been present when cough peak flow was measured. In comparable studies, a variety of measurement apparatus have been used, including different peak flow meters ${ }^{13,14,16}$ or pneumotachographs ${ }^{12,17}$ connected to masks or lip seals. All these methods are recommended by The British Thoracic Society to measure cough peak flow. ${ }^{4}$ It is plausible that dislodgement of the mask that led to a poor seal during assisted coughing may have occurred in our study. However, to minimize any potential measurement error, an investigator held the mask firmly in place during each measurement, with the sole role of ensuring a good mask seal during all maneuvers.

A limitation of this study was that the order of the interventions was not randomized but was standardized, with the application of a manually assisted cough performed by a physiotherapist first, then the application of manually assisted coughs performed by a parent or a caregiver second. The order of the interventions was not randomized because a key element of the study protocol was to train the parents to perform a manually assisted cough. An essential part of the teaching process was for a physiotherapist to demonstrate a manually assisted cough technique to the parents and caregivers. It made sense to standardize the number of coughs per child by measuring the physiotherapist's application of a manually assisted cough at the time of demonstration to the parent or caregiver.

A final limitation of this study was that only 20 min was allocated to teach a manually assisted cough and to collect data for each child and parent or caregiver because the study was conducted in a normal neuromuscular clinic, where regular care was provided in coordination with other health professionals. Given this time constraint, the parents and caregivers were not able to practice in their home environment, and the results, therefore, reflected only one encounter in time.

Despite the limitations and bias of this study, there were several reasons why the physiotherapists and the parents and caregivers were not able to improve the child's cough peak flow. One possible reason was that many of the parents and caregivers and children who participated in the study were learning this technique for the first time. In a retrospective medical record review, it was found that only 12 parents and caregivers and their children had previously been taught how to perform a manually assisted cough. Due to the limited detail from the retrospective review, it was not clear whether the parent or caregiver who was assessed was the same parent or caregiver who had previously received training. It was also not clear whether these parents and caregivers had continued to perform manually assisted coughs in the home environment. With several alternative manually assisted cough tech- niques, it was also unknown which technique had potentially been previously taught. Our study did not compare the outcomes of parents and caregivers who had previously learned the technique with those who had not, for the reasons discussed above.

Another possible reason may be that the 16 children who had not previously learned a manually assisted cough technique did not have time to adjust to the idea of a new technique because they were recruited on the day of their participation. Therefore, the children's coordination and cooperation with the techniques may have been limited. Because a manually assisted cough technique involves cooperation and coordination between the child and the individual applying the manually assisted cough, ${ }^{8}$ it can be argued that a child's familiarization with the technique and subsequent confidence may affect his or her coordination, and, therefore, the effectiveness of the technique when applied by a health-care provider or by a parent or a caregiver. In future studies, it would be prudent to ascertain what previous experience parents and caregivers may have had before beginning the study.

In addition, parents and caregivers were allowed a maximum of 3 practice efforts in a single training session before their technique was measured. The rationale for 3 practice efforts was to standardize the teaching process and potentially prevent the child from fatiguing. However, a single training session may not have allowed enough opportunity to practice to enable the parents and caregivers and the children to familiarize and consolidate the technique adequately. Furthermore, the amount of training for a health-care provider to effectively administer a manually assisted cough has not been quantified. Accordingly, it is not known the amount of training required before a parent can potentially effectively administer a manually assisted cough. Given that the physiotherapists were well practiced with the technique and were still unable to achieve a significant improvement in the subjects' cough peak flow, it is certainly worthwhile considering future research into the training requirements of healthcare providers and of parents and caregivers to effectively perform this intervention.

Our study specifically assessed the effect of a manually assisted cough as a stand-alone technique and found that neither health-care providers, nor parents and caregivers were effective in increasing the cough effectiveness of children with a range of neuromuscular diseases. In light of the results of our study, considerations should be given to the findings of several other studies ${ }^{11-13,15-18}$ that looked at manually assisted cough in conjunction with other inspiratory and expiratory cough augmentation. These other techniques included but were not limited to mechanical insufflation-exsufflation and lung volume recruitment, or breath-stacking before a manually assisted cough. Because this was the first study, to our knowl- 


\section{Teaching Manually Assisted Cough to Caregivers}

edge, to look at parents' and caregivers' ability to perform any technique aimed at improving cough peak flow, further research into parents' and caregivers' ability to successfully apply the other techniques described in the literature is warranted.

\section{Conclusions}

This study showed that parents and caregivers of children with NMD were not able to effectively apply a manually assisted cough to a child with neuromuscular weakness after a single training session with a health-care provider. These findings indicated single training sessions for parents and caregivers on how to perform a manually assisted cough were insufficient, and this should lead healthcare providers to assess the effectiveness of their interventions more closely. This study also identified that the literature about the effectiveness of a manually assisted cough performed by health-care providers in the population studied show varied results, with some studies that indicate a large increase in cough peak flow and other studies that show minor increases that are not likely to be clinically important. Further research is warranted to guide recommendations on how best to equip parents and caregivers with the skills to help manage children with NMD.

\section{ACKNOWLEDGMENTS}

We thank all the children and parents who agreed to partake in this study and the staff from the Sydney Children's Hospital Network. We thank Associate Professor Mark Elkins and Dr Jennifer K Peat, consultant biostatistician, for their guidance and expertise in the statistical analysis.

\section{REFERENCES}

1. De Vivo DC, Darras BT, Ryan MM, Roydon Jones H. Introduction: Historical Perspectives. In Darras BT, Royden Jones H, Ryan MM, De Vivo DC. Neuromuscular Disorders of Infancy, Childhood and Adolescence: A Clinician's Approach, 2nd edition. London, UK: Academic Press; 2015:3-15.

2. Bushby K, Finkel R, Birnkrant DJ, Case LE, Clemens PR, Cripe L, et al; DMD Care Considerations Working Group. Diagnosis and management of Duchenne muscular dystrophy, part 1: diagnosis, and pharmacological and psychosocial management. Lancet Neurol 2010; 9(1):77-93.
3. Phillips R, Edwards E, McNamara D, Reed P. Does use of the Cough Assist Machine reduce respiratory morbidity for children with neuromuscular disease? N Z J Physiother 2014;42(3):126-132.

4. Hull J, Aniapravan R, Chan E, Chatwin M, Forton J, Gallagher J, et al. British Thoracic Society guideline for respiratory management of children with neuromuscular weakness. Thorax 2012;67(Suppl 1):i1-i40.

5. Seddon PC, Khan Y. Respiratory problems in children with neurological impairment. Arch Dis Child 2003;88(1):75-78.

6. Gauld LM, Boynton A. Relationship between peak cough flow and spirometry in Duchenne muscular dystrophy. Pediatr Pulmonol 2005; 39(5):457-460.

7. Bianchi C, Baiardi P. Cough peak flows: standard values for children and adolescents. Am J Phys Med Rehabil 2008;87(6):461-467.

8. Bach JR. Update and perspective on noninvasive respiratory muscle aids. Part 2: The expiratory aids. Chest 1994;105(5):1538-1544.

9. Avena Kde M, Duarte AC, Cravo SL, Sologuren MJ, Gastaldi AC. Effects of manually assisted coughing on respiratory mechanics in patients requiring full ventilatory support. J Bras Pneumol 2008; 34(6):380-386.

10. Field AP. Discovering Statistics Using SPSS, 2nd edition. London, UK: Sage Publications Ltd; 2005.

11. Brito MF, Moreira GA, Pradella-Hallinan M, Tufik S. Air stacking and chest compression increase peak cough flow in patients with Duchenne muscular dystrophy. J Bras Pneumol 2009;35(10):973979.

12. Sivasothy P, Brown L, Smith IE, Shneerson JM. Effect of manually assisted cough and mechanical insufflation on cough flow of normal subjects, patients with chronic obstructive pulmonary disease (COPD), and patients with respiratory muscle weakness. Thorax 2001;56(6):438-444.

13. Ishikawa $\mathrm{Y}$, Bach JR, Komaroff E, Miura T, Jackson-Parekh R. Cough augmentation in Duchenne muscular dystrophy. Am J Phys Med Rehabil 2008;87(9):726-730.

14. Kim SM, Choi WA, Won YH, Kang SW. A Comparison of Cough Assistance Techniques in Patients with Respiratory Muscle Weakness. Yonsei Med J 2016;57(6):1488-1493.

15. Kang SW, Kang YS, Sohn HS, Park JH, Moon JH. Respiratory Muscle Strength and Cough Capacity in Patients with Duchenne Muscular Dystrophy. Yonsei Med J 2006;47(2):184-190.

16. Kang SW, Kang YS, Moon JH, Yoo TW. Assisted Cough and Pulmonary Compliance in Patients with Duchenne Muscular Dystrophy. Yonsei Med J 2005;46(2):233-238.

17. Chatwin M, Ross E, Hart N, Nickol AH, Polkey MI, Simonds AK. Cough augmentation with mechanical insufflation/exsufflation in patients with neuromuscular weakness. Eur Respir J 2003;21(3):502508 .

18. Toussaint M, Boitano LJ, Gathot V, Steenss M, Soudon P. Limits of effective cough-augmentation techniques in patients with neuromuscular disease. Respir Care 2009;54(3):359-366. 